\title{
On the global minimizers of a nonlocal isoperimetric problem in two dimensions
}

\author{
Peter STERnberg AND IHSAN TOPALOGLU \\ Department of Mathematics, Indiana University, Bloomington, IN 47405, USA \\ E-mail: sternber@indiana.edu,itopalog@indiana.edu
}

[Received 21 April 2010 and in revised form 8 October 2010]

\begin{abstract}
We analyze the minimization of a nonlocal isoperimetric problem (NLIP) posed on the flat 2-torus. After establishing regularity of the free boundary of minimizers, we show that when the parameter controlling the influence of the nonlocality is small, there is an interval of values for the mass constraint such that the global minimizer is exactly lamellar, that is, the free boundary consists of two parallel lines. In other words, in this parameter regime, the global minimizer of the $2 \mathrm{~d}$ (NLIP) coincides with the global minimizer of the local periodic isoperimetric problem.
\end{abstract}

\section{Introduction}

The nonlocal isoperimetric problem (NLIP) is given by

$$
\operatorname{minimize} \quad E_{\gamma}(u):=\frac{1}{2} \int_{\mathbb{T}^{2}}|\nabla u|+\gamma \int_{\mathbb{T}^{2}}|\nabla v|^{2} \mathrm{~d} x,
$$

over all $u \in B V\left(\mathbb{T}^{2},\{ \pm 1\}\right)$ satisfying

$$
\int_{\mathbb{T}^{2}} u \mathrm{~d} x=m
$$

and $v$ satisfying

$$
-\Delta v=u-m \quad \text { in } \mathbb{T}^{2} \quad \text { with } \quad \int_{\mathbb{T}^{2}} v \mathrm{~d} x=0 .
$$

Here $\mathbb{T}^{2}$ is the flat 2-torus and the total variation in the first term in $E_{\gamma}$ computes the perimeter of the set $\{x: u(x)=1\}$. For an interval of $m$-values containing $m=0$ and for $\gamma$ small, we will show that the global minimizer is lamellar, that is, the set $\{x: u(x)=1\}$ is simply a strip.

The problem (NLIP) arises, up to a constant factor, as the $\Gamma$-limit as $\varepsilon \rightarrow 0$ of the well-studied Ohta-Kawasaki sequence of functionals $E_{\varepsilon, \gamma}$ which model microphase separation of diblock copolymers, [1, 18]:

$E_{\varepsilon, \gamma}(u):= \begin{cases}\int_{\mathbb{T}^{2}}\left(\frac{\varepsilon}{2}|\nabla u|^{2}+\frac{\left(1-u^{2}\right)^{2}}{4 \varepsilon}+\gamma|\nabla v|^{2}\right) \mathrm{d} x & \text { if } u \in H^{1}\left(\mathbb{T}^{2}\right) \text { and } \int_{\mathbb{T}^{2}} u \mathrm{~d} x=m, \\ +\infty & \text { otherwise, }\end{cases}$

where again $v$ satisfies (1.2). There is an extensive literature exploring the energy landscape for $E_{\varepsilon, \gamma}$ in two and three dimensions, whether posed on the flat torus (i.e. with periodic boundary conditions) or on a general domain with homogeneous Neumann data (cf. e.g. [3, 20,-24]). The picture is quite 
rich and complicated, with the diffuse interface sometimes bounding one or more strips, wriggled strips, discs or ovals.

Much the same richness exists for the energy landscape of (NLIP). As such, independent of its connection to Ohta-Kawasaki, (NLIP) attracts interest as a rather canonical nonlocal perturbation of the classical isoperimetric problem. Indeed, as a model for pattern formation, (NLIP) sets up a basic competition between low surface area (the perimeter term) and high oscillation (the nonlocal term).

In three dimensions, computations reveal a wide array of stable critical points, with the free boundary $\partial\{x: u(x)=1\}$ consisting of one or more pairs of parallel planes, one or more spheres, cylinders or even hypersurfaces resembling more exotic triply periodic constant mean curvature surfaces such as gyroids, depending on where in the $(m, \gamma)$ parameter space one looks. With few exceptions, however, rigorous proofs of stability for particular patterns are rare (cf. e.g. [2, 25--27]), and to our knowledge, there are no proofs of global or even local minimality of specific critical points. In this regard, we mention the interesting investigation of [19], in which the authors seek to show that a lamellar (striped) pattern minimizes energy for a slightly different model related to diblock copolymers. Commenting on the inherent difficulty in picking out such a pattern as the "winner" in an energy landscape full of locally minimizing competitors, the authors of [19] remark, "...when comparing a striped pattern with arbitrary multidimensional patterns we know of no rigorous results, for any system." We also note the recent work [17] on a characterization of minimizers in a related model including screened Coulomb interaction in the setting of small volume fraction. There the author shows that minimizers form a collection of nearly identical circular droplets.

Here we have chosen to focus on the two-dimensional setting of (NLIP) with $\gamma$ small in order to present what is perhaps the first rigorous proof that a particular pattern is globally minimizing. To be more specific, fixing any $m \in(-1,1)$ let us define the lamellar function $u_{L}: \mathbb{T}^{2} \rightarrow \mathbb{R}$ whose phase $\{x: u(x)=1\}$ occupies in $[0,1] \times[0,1]$ a single vertical strip given by

$$
u_{L}\left(x_{1}, x_{2}\right)= \begin{cases}1 & \text { if } x_{1} \in(1 / 4-m / 2,3 / 4) \\ -1 & \text { if } x_{1} \notin(1 / 4-m / 2,3 / 4)\end{cases}
$$

One easily checks that $u_{L}$ is a critical point of $E_{\gamma}$ for all $\gamma$. (See (2.3) for the precise characterization of criticality.) Furthermore, for small $\gamma$ it is stable in the sense of nonnegative second variation, as shown in [5, Proposition 3.5], and it is unstable for larger $\gamma$ (cf. [5, Proposition 3.6], and [22]). However, in this article we go further to establish the global minimality of this lamellar critical point for small $\gamma$ when $m$ lies in the interval $(2 / \pi-1,1-2 / \pi)$. For $\gamma=0$, we note that the problem reduces to the well-known (local) periodic isoperimetric problem (cf. [4] 10]):

$$
\begin{gathered}
\text { minimize } E_{0}(u):=\frac{1}{2} \int_{\mathbb{T}^{2}}|\nabla u| \\
\text { over all } u \in B V\left(\mathbb{T}^{2},\{ \pm 1\}\right) \text { subject to } \int_{\mathbb{T}^{2}} u \mathrm{~d} x=m .
\end{gathered}
$$

For this problem the lamellar critical point $u_{L}$ is known to be the global minimizer for $m \in$ $(2 / \pi-1,1-2 / \pi)$ in two dimensions (cf. [12]). Thus, the restriction on the mass $m$ in our result is inherited from the global minimality of the strip in the local periodic isoperimetric problem, and is surely a necessary condition for global minimality with respect to $E_{\gamma}$ for $\gamma>0$. In light of the $\Gamma$-convergence of $E_{\gamma}$ to $E_{0}$ it is easy to see that the minimizer of $E_{\gamma}$, say $u_{\gamma}$, is close to $u_{L}$ when 
$\gamma$ is small, as shown in Proposition 3.1 We emphasize, however, that our main result, Theorem 3.3. says more, that is, $u_{\gamma} \equiv u_{L}$.

Our argument requires the regularity of minimizers to (NLIP). This regularity will be established in the next section. Though for our purposes, we only require regularity for global minimizers in the 2-torus, we establish the regularity result below, Proposition 2.1. for local minimizers in general $n$-dimensional domains since the regularity of (NLIP) is needed in other investigations such as [5] and we hope it will be useful to other authors. Given the well-developed regularity theory for areaminimizing sets (cf. e.g. [8] ), the issue here is to obtain a good estimate on the "excess-like" quantity that measures how far a set is from minimizing perimeter in a ball in terms of the radius of that ball (cf. 2.15). We show that even with the inclusion of the nonlocal term, it is still possible to obtain an estimate of order $\mathcal{O}\left(R^{n-1+\varepsilon}\right)$ in a ball of radius $R$, hence allowing us to invoke the standard theory.

The proof of the main theorem appears in the third section. Our method for proving the global minimality of the lamellar critical point $u_{L}$ consists of several steps. First, we must "corral" any reasonable competitor by showing that if the set where it equals one is not uniformly close to that of $u_{L}$, then necessarily it has too much perimeter. Such a corralling step often occurs when working with sets of finite perimeter in the $L^{1}$-topology (cf. e.g. [15, 29]), but here the argument is in some ways more subtle due to the nonlocal term in the energy which prefers multi-component competitors. Next, we show that competitors that are uniformly close are in fact $C^{2}$-close. Finally, we exploit the known stability of $u_{L}$ in the sense of second variation to eliminate competitors that are $C^{2}$-close. For the scenario of converting stability to either local or global minimality in the context of (local) volume-constrained least area problems, we should mention the works [9, 16].

\section{Regularity}

In this section, we establish the regularity of the set $\partial\{x: u(x)=1\}$ for any local minimizer $u$ of the $n$-dimensional version of (NLIP), namely

$$
\text { locally minimize } \quad \mathcal{E}_{\gamma}(A):=\int_{U}\left|\nabla \chi_{A}\right|+\gamma \int_{U}\left|\nabla v_{A}\right|^{2} \mathrm{~d} x
$$

over $A \subset U$ such that $|A|=(1+m) / 2$, where $-\Delta v_{A}=u_{A}-m$ and $u_{A}:=\chi_{A}-\chi_{A^{c}}$. Here $U$ can denote either any bounded domain in $\mathbb{R}^{n}$ or the flat $n$-torus $\mathbb{T}^{n}$.

We note that the formulation above is of course equivalent to (NLIP) given by (1.1). We will phrase the regularity result in terms of $L^{1}$-local minimizers, by which we mean any set $\Omega \subset U$ of finite perimeter such that

$$
\mathcal{E}_{\gamma}(\Omega) \leqslant \mathcal{E}_{\gamma}(A) \text { provided } \int_{U}\left|\chi_{\Omega}-\chi_{A}\right| \mathrm{d} x<\delta
$$

for some $\delta>0$.

PRoposition 2.1 Let $\Omega$ be an $L^{1}$-local minimizer of $(2.1)$ and denote by $\partial^{*} \Omega$ the reduced boundary of $\Omega$. Then $\partial^{*} \Omega \cap U$ is of class $C^{3, \alpha}$ for some $\alpha \in(0,1)$ and $\mathcal{H}^{s}\left[\left(\partial \Omega \backslash \partial^{*} \Omega\right) \cap U\right]=0$ for every nonnegative $s$ such that $s>n-8$. Furthermore, the solution $v_{\Omega}$ to $-\Delta v_{\Omega}=u_{\Omega}-m$ is of class $C^{1, \alpha}$ and one has the criticality condition

$$
(n-1) H(x)+4 \gamma v_{\Omega}(x)=\lambda
$$

for any $x \in \partial^{*} \Omega \cap U$ where $H$ denotes the mean curvature of $\partial \Omega, \mathcal{H}^{s}$ denotes $s$-dimensional Hausdorff measure and $\lambda$ is a constant. 
REMARK 2.2 Condition [2.3) as well as the second variation of $\mathcal{E}_{\gamma}$ are derived in [5] under the assumption of regularity of the free boundary.

REMARK 2.3 In Section 7.2 of [28], the authors establish $C^{3, \alpha}$ regularity of the reduced boundary of critical points of $\mathcal{E}_{\gamma}$ that arise in the limit $\varepsilon \rightarrow 0$ of critical points of the Ohta-Kawasaki energy $E_{\varepsilon, \gamma}$ given in 1.3.

Proof of Proposition 2.1 Let $\Omega$ be an $L^{1}$-local minimizer of $\mathcal{E}_{\gamma}$ and let $x_{0}$ be any point of $\partial \Omega \cap U$. Then let $D \subset \subset U$ be such that $x_{0} \notin \bar{D}$ and

$$
\int_{D}\left|\nabla \chi_{\Omega}\right|>0
$$

By [7. Lemma 2.1], there exist positive constants $k_{0}$ and $l_{0}$, depending only on $D$ and $D \cap \Omega$, such that for every $k$ with $|k|<k_{0}$ there exists a set $F$, with $F=\Omega$ outside $D$, and

$$
\begin{aligned}
|F| & =|\Omega|+k, \\
\int_{D}\left|\nabla \chi_{F}\right| & \leqslant \int_{D}\left|\nabla \chi_{\Omega}\right|+l_{0}|k|, \\
\int_{D}\left|\chi_{F}-\chi_{\Omega}\right| \mathrm{d} x & \leqslant l_{0}|k| \int_{D}\left|\nabla \chi_{\Omega}\right| .
\end{aligned}
$$

Here $|F|$ is the $n$-dimensional Lebesgue measure of the set $F$.

Fix $R>0$ such that

$$
\omega_{n} R^{n}<k_{0}, \quad\left(1+l_{0} \int_{D}\left|\nabla \chi_{\Omega}\right|\right) \omega_{n} R^{n}<\delta, \quad \bar{B}_{R}\left(x_{0}\right) \cap \bar{D}=\emptyset,
$$

where $\omega_{n}$ is the measure of the unit $n$-ball and $\delta$ comes from 2.2). Moreover, let $\tilde{F}$ minimize perimeter in $B_{R}\left(x_{0}\right)$ subject to the boundary values of $\Omega$, i.e.

$$
\int_{B_{R}\left(x_{0}\right)}\left|\nabla \chi_{\tilde{F}}\right| \leqslant \int_{B_{R}\left(x_{0}\right)}\left|\nabla \chi_{F}\right|
$$

for all $F$ such that $F \backslash B_{R}\left(x_{0}\right)=\Omega \backslash B_{R}\left(x_{0}\right)$. Without loss of generality, we can assume that $\left|\tilde{F} \cap B_{R}\left(x_{0}\right)\right| \leqslant\left|\Omega \cap B_{R}\left(x_{0}\right)\right|$. Since $\tilde{F} \cap \bar{D}=\Omega \cap \bar{D}$, we can use the same $k_{0}$ and $l_{0}$ as above with $\tilde{F}$ replacing $\Omega$ in $(2.4)$. Hence, for $k:=|\Omega|-|\tilde{F}| \leqslant \omega_{n} R^{n}<k_{0}$, there exists a set $G$, with $G=\tilde{F}$ outside $D$, and

$$
\begin{gathered}
|G|=|\Omega|=m, \\
\int_{D}\left|\nabla \chi_{G}\right| \leqslant \int_{D}\left|\nabla \chi_{\tilde{F}}\right|+C R^{n}, \\
\int_{U}\left|\chi_{G}-\chi_{\Omega}\right| \mathrm{d} x \leqslant C_{0} R^{n}<\delta,
\end{gathered}
$$

where the last condition follows from 2.5) with $C_{0}:=\left(1+l_{0} \int_{D}\left|\nabla \chi_{\Omega}\right|\right) \omega_{n}$.

Since by 2.6, $G$ is a competitor in $(2.1]$, we have

$$
\int_{U}\left|\nabla \chi_{\Omega}\right|+\gamma \int_{U}\left|\nabla v_{\Omega}\right|^{2} \mathrm{~d} x \leqslant \int_{U}\left|\nabla \chi_{G}\right|+\gamma \int_{U}\left|\nabla v_{G}\right|^{2} \mathrm{~d} x
$$


Thus, using the facts $\tilde{F} \backslash B_{R}\left(x_{0}\right)=\Omega \backslash B_{R}\left(x_{0}\right)$ and $G \backslash D=\tilde{F} \backslash D$, along with 2.7), the inequality (2.9) becomes

$$
\begin{aligned}
& \int_{U \backslash\left(D \cup B_{R}\left(x_{0}\right)\right)}\left|\nabla \chi_{\Omega}\right|+\int_{D}\left|\nabla \chi_{\tilde{F}}\right|+\int_{B_{R}\left(x_{0}\right)}\left|\nabla \chi_{\Omega}\right|+\gamma \int_{U}\left|\nabla v_{\Omega}\right|^{2} \mathrm{~d} x \\
& \leqslant \int_{U \backslash\left(D \cup B_{R}\left(x_{0}\right)\right)}\left|\nabla \chi_{G}\right|+\int_{D}\left|\nabla \chi_{G}\right|+\int_{B_{R}\left(x_{0}\right)}\left|\nabla \chi_{G}\right|+\gamma \int_{U}\left|\nabla v_{G}\right|^{2} \mathrm{~d} x \\
&=\int_{U \backslash\left(D \cup B_{R}\left(x_{0}\right)\right)}\left|\nabla \chi_{\Omega}\right|+\int_{D}\left|\nabla \chi_{G}\right|+\int_{B_{R}\left(x_{0}\right)}\left|\nabla \chi_{\tilde{F}}\right|+\gamma \int_{U}\left|\nabla v_{G}\right|^{2} \mathrm{~d} x \\
& \leqslant \int_{U \backslash\left(D \cup B_{R}\left(x_{0}\right)\right)}\left|\nabla \chi_{\Omega}\right|+\int_{D}\left|\nabla \chi_{\tilde{F}}\right|+\int_{B_{R}\left(x_{0}\right)}\left|\nabla \chi_{\tilde{F}}\right|+\gamma \int_{U}\left|\nabla v_{G}\right|^{2} \mathrm{~d} x+C R^{n} .
\end{aligned}
$$

Hence, we get

$$
\int_{B_{R}\left(x_{0}\right)}\left|\nabla \chi_{\Omega}\right|-\int_{B_{R}\left(x_{0}\right)}\left|\nabla \chi_{\tilde{F}}\right| \leqslant \gamma \int_{U}\left|\nabla v_{G}\right|^{2} \mathrm{~d} x-\gamma \int_{U}\left|\nabla v_{\Omega}\right|^{2} \mathrm{~d} x+C R^{n} .
$$

Now we estimate the nonlocal parts on the right-hand side of 2.10). To this end, let $w:=v_{\Omega}-v_{G}$. Then $-\Delta w=u_{\Omega}-u_{G}$ with $\int_{U} w \mathrm{~d} x=0$, where $\left|u_{\Omega}-u_{G}\right|$ is equal to zero in $U \backslash\left(B_{R}\left(x_{0}\right) \cup D\right)$ and is bounded by 2 in $B_{R}\left(x_{0}\right) \cup D$. Hence for any $p \geqslant 1$ we have

$$
\left\|u_{\Omega}-u_{G}\right\|_{L^{p}(U)} \leqslant C R^{n / p},
$$

through an appeal to 2.8 .

We take

$$
p=\kappa \frac{n}{n-1},
$$

where $\kappa$ is less than but as close as needed to 1 so that $1<p<n /(n-1)$, and apply the CalderónZygmund inequality (cf. e.g. [6, Chapter 9] or [11] for the periodic case and [31, Chapter 2] for the Neumann case),

$$
\|w\|_{W^{2, p}(U)} \leqslant C\left\|u_{\Omega}-u_{G}\right\|_{L^{p}(U)} .
$$

Then by the Sobolev imbedding theorem, for $q=\kappa n /(n-(1+\kappa))$, along with the Hölder and Poincaré inequalities we get

$$
\|w\|_{L^{1}(U)} \leqslant C\|w\|_{L^{q}(U)} \leqslant C\|\nabla w\|_{L^{q}(U)} \leqslant C\|w\|_{W^{2, p}(U)} .
$$

Note that, as $\kappa \rightarrow 1, \kappa n /(n-(1+\kappa))$ approaches $n /(n-2)$, so in particular $q>1$. Thus, by combining 2.11 2.13 we obtain

$$
\|w\|_{L^{1}(U)} \leqslant C R^{(n-1) / \kappa},
$$

or in other words, since $\kappa<1$, we have

$$
\int_{U}\left|v_{\Omega}-v_{G}\right| \mathrm{d} x=\int_{U}|w| \mathrm{d} x \leqslant C R^{n-1+\varepsilon}
$$

for some $\varepsilon>0$. (We should perhaps note that for the case $n=2$, the desired inequality $\|w\|_{L^{1}(U)} \leqslant$ $C\left\|u_{\Omega}-u_{G}\right\|_{L^{p}(U)}$ is a simple consequence of Poincaré and Hölder, without even an appeal to Calderón-Zygmund, since then $H^{1}$ imbeds continuously into any $L^{p}$ with $p<\infty$.) 
Now, using (2.8), 2.14) and integration by parts, we obtain the following bound on the difference of the nonlocal parts:

$$
\int_{U}\left|\nabla v_{G}\right|^{2} \mathrm{~d} x-\int_{U}\left|\nabla v_{\Omega}\right|^{2} \mathrm{~d} x \leqslant \int_{U}\left|u_{\Omega}-u_{G}\right|\left|v_{G}\right| \mathrm{d} x+\int_{U}\left|v_{\Omega}-v_{G}\right|\left|u_{\Omega}\right| \mathrm{d} x \leqslant C R^{n-1+\varepsilon} .
$$

Returning to 2.10 , this implies that

$$
\int_{B_{R}\left(x_{0}\right)}\left|\nabla \chi_{\Omega}\right|-\int_{B_{R}\left(x_{0}\right)}\left|\nabla \chi_{\tilde{F}}\right| \leqslant C R^{n-1+\varepsilon} .
$$

Property (2.15) states that the boundary of the set $\Omega$ is almost area-minimizing in any ball. With this property in hand, the results of [14, 30] apply, and we can conclude that $\partial^{*} \Omega \cap U$ is of class $C^{1, \alpha}$, with $\mathcal{H}^{s}\left[\left(\partial \Omega \backslash \partial^{*} \Omega\right) \cap U\right]=0$ for every $s>n-8$.

Next we note that since $\left\|u_{\Omega}\right\|_{L^{\infty}}=1$, we have a bound on $\left\|v_{\Omega}\right\|_{W^{2, p}}$ for any $p<\infty$ by elliptic regularity. Hence, by the Sobolev imbedding theorem we get a bound on $\left\|v_{\Omega}\right\|_{C^{1, \alpha}}$ for any $\alpha<1$ as well.

With $C^{1, \alpha}$ regularity of the reduced boundary in hand, one can locally describe $\partial^{*} \Omega$ in nonparametric form, as say, the graph of a $C^{1, \alpha}$ function $\Phi$ on a ball $B \subset \mathbb{R}^{n-1}$. Then one can compute the first variation of $\mathcal{E}_{\gamma}$ to find that $\Phi$ weakly solves

$$
(n-1) \nabla \cdot\left(\frac{\nabla \Phi\left(x^{\prime}\right)}{\sqrt{1+\left|\nabla \Phi\left(x^{\prime}\right)\right|^{2}}}\right)=-4 \gamma v_{\Omega}\left(x^{\prime}, \Phi\left(x^{\prime}\right)\right)+\lambda \quad \text { for } x^{\prime} \in B
$$

where $\lambda$ appears as a Lagrange multiplier due to the volume constraint. Since the right-hand side is of class $C^{1, \alpha}$, it follows from standard elliptic theory that $\Phi$ is in fact of class $C^{3, \alpha}$ and so, in particular, 2.3. holds classically.

\section{Global minimizers of $E_{\gamma}$}

In this section, returning to the two-dimensional periodic setting, we will prove our main result, namely that for $\gamma$ sufficiently small, the global minimizer $u_{\gamma}$ of $E_{\gamma}$ coincides with $u_{L}$, the lamellar critical point.

Here, emphasizing the $\gamma$ dependence, we want to note that in two dimensions the criticality condition (2.3) obtained in Proposition 2.1 becomes

$$
H_{\gamma}(x)+4 \gamma v_{\gamma}(x)=\lambda_{\gamma}
$$

for all $x \in \partial\left\{x: u_{\gamma}(x)=1\right\}$.

We can immediately conclude that any sequence of minimizers $\left\{u_{\gamma}\right\}$ of (1.1) converges, after perhaps a translation, to the lamellar minimizer $u_{L}$ given by (1.4) of $E_{0}$ defined in (1.5).

PROPOSITION 3.1 For any $m$ satisfying $|m|<1-2 / \pi$, let $\left\{u_{\gamma}\right\}_{\gamma} \geqslant 0$ be a sequence of minimizers of $E_{\gamma}$. Then after perhaps a translation,

$$
u_{\gamma} \rightarrow u_{L} \quad \text { in } L^{1}\left(\mathbb{T}^{2}\right) \text { as } \gamma \rightarrow 0 .
$$


Proof. Since a uniform bound $E_{\gamma}\left(u_{\gamma}\right)<C$ is immediate in light of the minimality of $u_{\gamma}$, one obtains a uniform $B V$-bound leading to convergence in $L^{1}$ of a subsequence. By the standard $\Gamma$ convergence argument, this limit must minimize $E_{0}$, the periodic isoperimetric problem. In two dimensions it is known that the global minimizer of $E_{0}$ is lamellar for $|m|<1-2 / \pi$, hence is equal to $u_{L}$ given by (1.4), after a translation (cf. [12, Section 7]).

With this in hand, one can also easily establish convergence of the functions $v_{\gamma}$ to $v_{L}$.

Proposition 3.2 For any minimizer $u_{\gamma}$ of $E_{\gamma}$, there is a value $\alpha \in(0,1)$ such that the corresponding solution $v_{\gamma}$ of $(1.2)$ is bounded in $C^{1, \alpha}$ independent of $\gamma$. Moreover, for a sequence of minimizers $\left\{u_{\gamma}\right\}_{\gamma} \geqslant 0$ of $E_{\gamma}$ satisfying 3.2 we have

$$
v_{\gamma} \rightarrow v_{L} \quad \text { in } H^{2}\left(\mathbb{T}^{2}\right)
$$

In particular,

$$
\int_{\mathbb{T}^{2}}\left|\nabla v_{\gamma}\right|^{2} \mathrm{~d} x \rightarrow \int_{\mathbb{T}^{2}}\left|\nabla v_{L}\right|^{2} \mathrm{~d} x \quad \text { as } \gamma \rightarrow 0 .
$$

Proof. Note that since $\left\|u_{\gamma}\right\|_{L^{\infty}}=1$, we have a $\gamma$-independent bound on $\left\|v_{\gamma}\right\|_{W^{2, p}}$ for any $p<\infty$ by elliptic regularity. Hence, by the Sobolev imbedding theorem we get a $\gamma$-independent bound on $\left\|v_{\gamma}\right\|_{C^{1, \alpha}}$ for some $\alpha<1$ as well. Similarly, $v_{L} \in W^{2, p}$.

Now, let $w_{\gamma}:=v_{\gamma}-v_{L}$ and $\phi_{\gamma}:=u_{\gamma}-u_{L}$. Note that $-\Delta w_{\gamma}=\phi_{\gamma}$ and $\phi_{\gamma} \rightarrow 0$ in $L^{p}$ for all $1 \leqslant p<\infty$ as $\gamma \rightarrow 0$, since $\phi_{\gamma} \rightarrow 0$ in $L^{1}$ by assumption and $\left\|\phi_{\gamma}\right\|_{L^{\infty}} \leqslant 2$.

Since $w_{\gamma}$ is periodic, through an integration by parts we obtain $\int_{\mathbb{T}^{2}}\left(\Delta w_{\gamma}\right)^{2} \mathrm{~d} x=$ $\int_{\mathbb{T}^{2}}\left|\nabla^{2} w_{\gamma}\right|^{2} \mathrm{~d} x$; hence, $\left\|\nabla^{2} w_{\gamma}\right\|_{L^{2}} \rightarrow 0$ as $\gamma \rightarrow 0$.

Then, since $\int_{\mathbb{T}^{2}} v_{\gamma} \mathrm{d} x=\int_{\mathbb{T}^{2}} v_{L} \mathrm{~d} x=0$, we find that $\int_{\mathbb{T}^{2}} w_{\gamma} \mathrm{d} x=0$, and so the Poincaré inequality, $\left\|w_{\gamma}\right\|_{L^{2}} \leqslant C\left\|\nabla w_{\gamma}\right\|_{L^{2}}$, applies. Integrating by parts and using Hölder's inequality, we obtain

$$
\left\|\nabla w_{\gamma}\right\|_{L^{2}}^{2}=\int_{\mathbb{T}^{2}} w_{\gamma} \phi_{\gamma} \mathrm{d} x \leqslant\left\|w_{\gamma}\right\|_{L^{2}}\left\|\phi_{\gamma}\right\|_{L^{2}} \leqslant C\left\|\nabla w_{\gamma}\right\|_{L^{2}}\left\|\phi_{\gamma}\right\|_{L^{2}}
$$

and so $\left\|w_{\gamma}\right\|_{L^{2}}$ and $\left\|\nabla w_{\gamma}\right\|_{L^{2}}$ also tend to zero as $\gamma \rightarrow 0$. Thus, we get $\left\|w_{\gamma}\right\|_{H^{2}} \rightarrow 0$.

We now state our main result:

THEOREM 3.3 Fix any $m$ such that $|m|<1-2 / \pi$. Then for small $\gamma>0$, the minimizers $\left\{u_{\gamma}\right\}$ of $E_{\gamma}$ are lamellar, that is, $u_{\gamma} \equiv u_{L}$ up to translation.

Proof. We will prove the theorem in several steps. For simplicity of presentation only, we will take $m=0$ in the proof. Throughout the proof, we denote by $S$ the strip $\left\{\left(x_{1}, x_{2}\right): 1 / 4<x_{1}<3 / 4,0<\right.$ $\left.x_{2}<1\right\}$ and by $\Omega_{\gamma}$ the set $\left\{x: u_{\gamma}(x)=1\right\}$.

Step 1. We first claim there cannot exist a sequence of components $S_{\gamma}^{1} \subset \Omega_{\gamma}$ whose area converges to zero as $\gamma \rightarrow 0$.

To this end, we write $\Omega_{\gamma}$ as a union of its connected components, i.e. $\Omega_{\gamma}=\bigcup_{j=1}^{N_{\gamma}} S_{\gamma}^{j}$. We first note that necessarily $N_{\gamma}<\infty$ since otherwise for fixed $\gamma$ there would have to exist a sequence of components of $\Omega_{\gamma}$ whose area (and perimeter) approach zero. This would be impossible in light of (3.1) and Proposition 3.2, which imply a ( $\gamma$-dependent) bound on the $L^{\infty}$-norm of the curvature $H_{\gamma}$ of $\partial \Omega_{\gamma}$.

Now we assume, by way of contradiction, that for a sequence of $\gamma$-values approaching zero, $\Omega_{\gamma}$ has a component, say $S_{\gamma}^{1}$, with $\left|S_{\gamma}^{1}\right| \rightarrow 0$. 
Define $S_{\gamma}:=\Omega_{\gamma} \backslash S_{\gamma}^{1}$. Then $\chi_{S_{\gamma}} \rightarrow \chi_{S}$ in $L^{1}$. Also, note that $\operatorname{Per}_{\mathbb{T}^{2}}\left(S_{\gamma}^{1}\right) \rightarrow 0$, for if not, that is, if say $c:=\liminf _{\gamma \rightarrow 0} \operatorname{Per}_{\mathbb{T}^{2}}\left(S_{\gamma}^{1}\right)$ with $c>0$, then since $\liminf \frac{1}{2} \operatorname{Per}_{\mathbb{T}^{2}}\left(S_{\gamma}\right) \geqslant \frac{1}{2} \operatorname{Per}_{\mathbb{T}^{2}}(S)=1$, we get

$$
\liminf _{\gamma \rightarrow 0} E_{\gamma}\left(u_{\gamma}\right) \geqslant \frac{2+c}{2}>1
$$

This contradicts the fact that $E_{\gamma}\left(u_{\gamma}\right) \rightarrow E_{0}\left(u_{L}\right)=\frac{1}{2} \operatorname{Per}_{\mathbb{T}^{2}}(S)=1$ by $\Gamma$-convergence.

Now, the regularity of $\partial \Omega_{\gamma}$ asserted in Proposition 2.1 and the fact that $\operatorname{Per}_{\mathbb{T}^{2}}\left(S_{\gamma}^{1}\right) \rightarrow 0$ imply that we can enclose $S_{\gamma}^{1}$ in a disk whose radius approaches zero with $\gamma$. Shrink the disk until it touches $\partial S_{\gamma}^{1}$ for the first time, denoting the radius of the shrunken disk by $r_{\gamma}$ and the point where the disk touches $\partial S_{\gamma}^{1}$ by $p_{\gamma}$. Then we have $H_{\gamma}\left(p_{\gamma}\right) \geqslant 1 / r_{\gamma}$ and so by evaluating the criticality condition 3.1 at $x=p_{\gamma}$, we see that $\lambda_{\gamma} \rightarrow \infty$ since $\left\|v_{\gamma}\right\|_{L^{\infty}}$ is bounded independent of $\gamma$ by Proposition 3.2 Returning to 3.1) for $x \neq p_{\gamma}$, we conclude that in fact $H_{\gamma}(x) \rightarrow \infty$ for all $x \in \partial \Omega_{\gamma}$. Moreover, since $H_{\gamma}\left(p_{\gamma}\right) \geqslant 1 / r_{\gamma}$, for $\gamma$ small enough we have, say, $H_{\gamma}(x) \geqslant 1 /\left(4 r_{\gamma}\right)$ for all $x \in \partial \Omega_{\gamma}$, so $S_{\gamma}^{j}$ is contained in a disk of radius $2 r_{\gamma}$ for each $j \in\left\{1, \ldots, N_{\gamma}\right\}$.

For a finer analysis, let $\rho_{\gamma}^{j}:=\operatorname{diam}\left(S_{\gamma}^{j}\right)$. Then $S_{\gamma}^{j}$ is contained in a disk of radius $\rho_{\gamma}^{j}$. Now, define $\rho_{\gamma}:=\min \left\{\rho_{\gamma}^{j}: j \in\left\{1, \ldots, N_{\gamma}\right\}\right\}$ so that

$$
\operatorname{Per}_{\mathbb{T}^{2}}\left(S_{\gamma}^{j}\right) \geqslant \rho_{\gamma}^{j} \geqslant \rho_{\gamma} .
$$

Then let the minimum $\rho_{\gamma}$ be attained at, say, $j=j_{0}$, i.e., $\rho_{\gamma}=\left|p_{\gamma}^{j_{0}}-q_{\gamma}^{j_{0}}\right|$ with $p_{\gamma}^{j_{0}}, q_{\gamma}^{j_{0}}$ on $\partial S_{\gamma}^{j_{0}}$. As $\rho_{\gamma}=\left|p_{\gamma}^{j_{0}}-q_{\gamma}^{j_{0}}\right|$ and $S_{\gamma}^{j_{0}}$ is contained in a disk of radius $\rho_{\gamma}$ which must be tangent to $\partial S_{\gamma}^{j_{0}}$, say, at $p_{\gamma}^{j_{0}}$, we see that $H_{\gamma}\left(p_{\gamma}^{j_{0}}\right) \geqslant 1 / \rho_{\gamma}$. Hence, using the $L^{\infty}$-bound on $v_{\gamma}$ and the criticality condition, we get

$$
\frac{1}{\rho_{\gamma}}-C_{\gamma} \leqslant H_{\gamma}\left(p_{\gamma}^{j_{0}}\right)+4 \gamma v_{\gamma}\left(p_{\gamma}^{j_{0}}\right)=\lambda_{\gamma}
$$

where $C_{\gamma}$ depends only on $\gamma$ and $\left\|v_{\gamma}\right\|_{L^{\infty}}$, and $C_{\gamma}$ is $\mathcal{O}(\gamma)$. Thus at any point $x \in \partial \Omega_{\gamma}$ we have

$$
H_{\gamma}(x)+C_{\gamma} \geqslant H_{\gamma}(x)+4 \gamma v_{\gamma}(x)=\lambda_{\gamma} \geqslant \frac{1}{\rho_{\gamma}}-C_{\gamma},
$$

and this gives

$$
H_{\gamma}(x) \geqslant \frac{1}{\rho_{\gamma}}-2 C_{\gamma} \geqslant \frac{1}{2 \rho_{\gamma}} .
$$

Thus for any $j \in\left\{1, \ldots, N_{\gamma}\right\}, S_{\gamma}^{j}$ is contained in a disk of radius $2 \rho_{\gamma}$. Using this fact we can find a lower bound on $N_{\gamma}$ depending on $\rho_{\gamma}$ as follows: Since $\frac{1}{2}=\left|\Omega_{\gamma}\right|=\sum_{j=1}^{N_{\gamma}}\left|S_{\gamma}^{j}\right| \leqslant \pi\left(2 \rho_{\gamma}\right)^{2} N_{\gamma}$, we get

$$
N_{\gamma} \geqslant \frac{1}{8 \pi \rho_{\gamma}^{2}} .
$$

This lower bound on $N_{\gamma}$ with 3.3 will then imply that

$$
\operatorname{Per}_{\mathbb{T}^{2}}\left(\Omega_{\gamma}\right)=\sum_{j=1}^{N_{\gamma}} \operatorname{Per}_{\mathbb{T}^{2}}\left(S_{\gamma}^{j}\right) \geqslant \rho_{\gamma} N_{\gamma} \geqslant \frac{1}{8 \pi \rho_{\gamma}} .
$$

Hence $\operatorname{Per}_{\mathbb{T}^{2}}\left(S_{\gamma}\right) \rightarrow \infty$ as $\gamma \rightarrow 0$, which contradicts the fact that $E_{\gamma}\left(u_{\gamma}\right) \rightarrow 1$. 
Here we want to remark that the above argument also shows that there cannot be a sequence of components of the complement of $\Omega_{\gamma}$ approaching zero in measure.

Step 2. Let us again express $\Omega_{\gamma}$ as a union of its connected components, namely, $\Omega_{\gamma}=\bigcup_{j=1}^{N_{\gamma}} S_{\gamma}^{j}$. We next claim that for some $j$, the component $S_{\gamma}^{j}$ has at least two noncontractible boundary components.

Note that if a component $S_{\gamma}^{j}$ has one noncontractible boundary component, then since the homology classes of boundaries of a connected, compact set sum up to zero, there would then have to exist at least one other noncontractible boundary component of $S_{\gamma}^{j}$. In light of this, to establish our claim it suffices to reach a contradiction by assuming that all boundary components of $\Omega_{\gamma}$ are contractible in $\mathbb{T}^{2}$.

Considering the lifts of the boundary components to the covering space, we see that they are disjoint closed curves in $\mathbb{R}^{2}$, say, $\alpha_{1}, \ldots, \alpha_{n}$. After removing from this collection any curve $\alpha_{i}$ which is contained in the interior of the set enclosed by any other curve $\alpha_{j}$, we can relist the remaining curves as $\alpha_{1}, \ldots, \alpha_{k}$, where $k \leqslant n$. It follows that all points not in the union of the interiors of $\alpha_{1}, \ldots, \alpha_{k}$ must either lie entirely in the lift of $\Omega_{\gamma}$ or else in the lift of $\Omega_{\gamma}^{c}$. If, for example, the exterior of the curves lies completely within $\Omega_{\gamma}^{c}$, then we have $\left|\Omega_{\gamma}\right| \leqslant \sum_{i=1}^{k} \mid$ int $\alpha_{i} \mid$; hence using the isoperimetric inequality in $\mathbb{R}^{2}$,

$$
\left.\operatorname{Per}_{\mathbb{T}^{2}}\left(\Omega_{\gamma}\right)\right)^{2} \geqslant \sum_{i=1}^{k} l^{2}\left(\alpha_{i}\right) \geqslant 4 \pi \sum_{i=1}^{k}\left|\operatorname{int} \alpha_{i}\right| \geqslant 4 \pi\left|\Omega_{\gamma}\right|,
$$

where int $\alpha_{i}$ denotes the interior of the set enclosed by $\alpha_{i}$ and $l\left(\alpha_{i}\right)$ denotes the length of the curve $\alpha_{i}$. Similarly, if the exterior of the curves $\alpha_{1}, \ldots, \alpha_{k}$ lies entirely in $\Omega_{\gamma}$, then $\left|\Omega_{\gamma}^{c}\right| \leqslant \sum_{i=1}^{k} \mid$ int $\alpha_{i} \mid$, and so in that case we would have

$$
\left(\operatorname{Per}_{\mathbb{T}^{2}}\left(\Omega_{\gamma}\right)\right)^{2}=\left(\operatorname{Per}_{\mathbb{T}^{2}}\left(\Omega_{\gamma}^{c}\right)\right)^{2} \geqslant \sum_{i=1}^{k} l^{2}\left(\alpha_{i}\right) \geqslant 4 \pi \sum_{i=1}^{k}\left|\operatorname{int} \alpha_{i}\right| \geqslant 4 \pi\left|\Omega_{\gamma}^{c}\right| .
$$

Thus we get

$$
\left.\operatorname{Per}_{\mathbb{T}^{2}}\left(\Omega_{\gamma}\right)\right)^{2} \geqslant 4 \pi \min \left\{\left|\Omega_{\gamma}\right|,\left|\Omega_{\gamma}^{c}\right|\right\}
$$

Hence,

$$
\liminf _{\gamma \rightarrow 0} \operatorname{Per}_{\mathbb{T}^{2}}\left(\Omega_{\gamma}\right) \geqslant \sqrt{2 \pi}>2,
$$

which gives a contradiction since $E_{\gamma}\left(u_{\gamma}\right) \rightarrow E_{0}\left(u_{L}\right)=1$. Here we have used $m=0$ to see that $\min \left\{\left|\Omega_{\gamma}\right|,\left|\Omega_{\gamma}^{c}\right|\right\}=1 / 2$. Note, however, that for any $m$ such that $|m|<1-2 / \pi$, one has $\min \left\{\left|\Omega_{\gamma}\right|,\left|\Omega_{\gamma}^{c}\right|\right\}>1 / \pi$, still yielding a contradiction. We point out that this is the only place in the proof where the restriction $|m|<1-2 / \pi$ is used.

Therefore we see that there exists $j \in\left\{1, \ldots, N_{\gamma}\right\}$ such that $S_{\gamma}^{j}$ has a boundary component which is not contractible in $\mathbb{T}^{2}$. Hence, it has at least two such boundary components, say $\Sigma_{1, \gamma}^{j}$ and $\Sigma_{2, \gamma}^{j}$.

Step 3. We claim that $\Sigma_{1, \gamma}^{j}$ and $\Sigma_{2, \gamma}^{j}$ are both connected arcs on the unit square with endpoints either of the form $(a, 0),(a, 1)$ or of the form $(0, a),(1, a)$ for some $a \in[0,1]$. Furthermore, we claim that $\Omega_{\gamma}$ consists of precisely one component with $\partial \Omega_{\gamma}=\Sigma_{1, \gamma}^{j} \cup \Sigma_{2, \gamma}^{j}$. 
To this end, suppose $\Sigma_{1, \gamma}^{j}$ meets the boundary of the unit square $[0,1] \times[0,1]$ at, say, $(a, 0)$ or $(0, a)$ for some $a \in[0,1]$. Then for it to be closed, its lift to the covering space must eventually pass through a point $(b, c) \in \mathbb{R}^{2}$ that differs from $(a, 0)$ or $(0, a)$ by an element of the lattice $\mathbb{Z}^{2}$, as $\mathbb{T}^{2} \cong \mathbb{R}^{2} / \mathbb{Z}^{2}$. The shortest possibility is of course either $(a, \pm 1)$ or $( \pm 1, a)$, corresponding to vertical or horizontal line segments. However, failing this, the next shortest possibility would be either $(a \pm 1, \pm 1)$ or $( \pm 1, a \pm 1)$ and on the torus such a geodesic has length $\sqrt{2}$. This then would imply that $\operatorname{Per}_{\mathbb{T}^{2}}\left(\Sigma_{1, \gamma}^{j}\right) \geqslant \sqrt{2}$; hence, $\operatorname{Per}_{\mathbb{T}^{2}}\left(\Sigma_{1, \gamma}^{j} \cup \Sigma_{2, \gamma}^{j}\right) \geqslant 1+\sqrt{2}$, contradicting the fact that $\operatorname{Per}_{\mathbb{T}^{2}}\left(\Omega_{\gamma}\right)$ must approach 2 as $\gamma \rightarrow 0$. This shows the first claim of Step 3 .

Next we note that for all $\gamma$ sufficiently small, $\Omega_{\gamma}$ cannot have other boundary components. If, for a sequence of $\gamma$-values approaching zero, $\partial \Omega_{\gamma}$ possessed another component with the corresponding sequence of perimeters having a positive limit, this would again result in $\liminf _{\gamma \rightarrow 0} \operatorname{Per}_{\mathbb{T}^{2}}\left(\Omega_{\gamma}\right)>2$, an impossibility. On the other hand, Step 1 precludes the possibility of such a sequence of perimeters having zero limit.

Therefore $\Omega_{\gamma}$ has only one component with exactly two boundary components $\Sigma_{1, \gamma}$ and $\Sigma_{2, \gamma}$ having endpoints either of the form $(a, 0)$ and $(a, 1)$ or of the form $(0, a)$ and $(1, a)$ for some $a \in[0,1]$.

Step 4. Now we will show that for some $C>0$ independent of $\gamma$, one has

$$
\left|H_{\gamma}(x)\right| \leqslant C \gamma
$$

for all $x \in \partial \Omega_{\gamma}$.

We first claim that the constant $\lambda_{\gamma}$ in (3.1) tends to zero as $\gamma \rightarrow 0$. To prove this claim, consider $\Sigma_{1, \gamma}$ as described above. Let $\theta_{\gamma}(s)$ denote the angle made between the tangent to $\Sigma_{1, \gamma}$ and the standard basis vector $(1,0)$, where $s$ denotes arc-length, and let $l_{\gamma}$ denote the length of $\Sigma_{1, \gamma}$. From the regularity of $\Sigma_{1, \gamma}$ and its simple description from Step 3, and since $s=0$ and $s=l_{\gamma}$ correspond to the same points on $\mathbb{T}^{2}$, the angles at the two endpoints, $\theta(0)$ and $\theta\left(l_{\gamma}\right)$, must agree. Then, since $H_{\gamma}=\mathrm{d} \theta_{\gamma} / \mathrm{d} s$, we can integrate (3.1) to see that

$$
4 \gamma \int_{0}^{l_{\gamma}} v_{\gamma} \mathrm{d} s=\lambda_{\gamma} l_{\gamma}
$$

Noting that $l_{\gamma} \geqslant 1$ and invoking the uniform $L^{\infty}$-bound on $v_{\gamma}$ from Proposition 3.2 , we see that $\lambda_{\gamma} \rightarrow 0$ as $\gamma \rightarrow 0$, and indeed $\lambda_{\gamma}=\mathcal{O}(\gamma)$. Returning to 3.1., this immediately yields 3.47.

Step 5. We next claim that each boundary component of $\Omega_{\gamma}$ is globally the graph of a function.

It is enough to show this for $\Sigma_{1, \gamma}$, the arguments for the other component being identical. Since we are taking the lamellar set $S=\left\{x \in \mathbb{T}^{2}: u_{L}(x)=1\right\}$ to have vertical boundary components, we see that by Step $3, \Sigma_{1, \gamma}$ passes through the points $(a, 0)$ and $(a, 1)$ because if it passed through $(0, a)$ and $(1, a)$ instead, while maintaining a curvature that is $\mathcal{O}(\gamma)$, this would contradict the fact that $\chi_{\Omega_{\gamma}} \rightarrow \chi_{S}$ in $L^{1}$.

Consider the vertical line segments $\{0\} \times(0,1)$ and $\{1\} \times(0,1)$ and slide them to the right and left, respectively, until one of the translates touches $\Sigma_{1, \gamma}$ for the first time at a point $X^{0}=$ $\left(x_{1}^{0}, x_{2}^{0}\right)$ inside $(0,1) \times(0,1)$. Since $\Sigma_{1, \gamma}$ is smooth, in a neighborhood of $X^{0}, \Sigma_{1, \gamma}$ can be expressed locally as the graph of a function, say $x_{2} \mapsto 3 / 4+f_{\gamma}\left(x_{2}\right)$. In other words for some $\delta>0$ the set $\left\{\left(3 / 4+f_{\gamma}\left(x_{2}\right), x_{2}\right): x_{2} \in\left(x_{2}^{0}-\delta, x_{2}^{0}+\delta\right)\right\}$ agrees with $\Sigma_{1, \gamma}$.

We claim that the graph of $3 / 4+f_{\gamma}\left(x_{2}\right)$ agrees with $\Sigma_{1, \gamma}$ for all $x_{2} \in[0,1]$; in other words, we will show that the domain of $f_{\gamma}$ can be extended to $[0,1]$. To establish this, let $\delta_{0}$ be the smallest 
positive number such that $\left|f_{\gamma}^{\prime}\left(x_{2}^{0}+\delta_{0}\right)\right| \geqslant 1$. If no such number exists, then we can extend the domain of $f_{\gamma}$ all the way to $x_{2}=1$. By the mean value theorem and the fact that $f_{\gamma}^{\prime}\left(x_{2}^{0}\right)=0$, we get

$$
\left|f_{\gamma}^{\prime \prime}(\xi)\right|=\left|\frac{f_{\gamma}^{\prime}\left(x_{2}^{0}+\delta_{0}\right)-f_{\gamma}^{\prime}\left(x_{2}^{0}\right)}{\delta_{0}}\right| \geqslant \frac{1}{\delta_{0}}>1
$$

for some $\xi \in\left(x_{2}^{0}, x_{2}^{0}+\delta_{0}\right)$. This gives

$$
\left|\frac{f_{\gamma}^{\prime \prime}(\xi)}{\left(1+\left(f_{\gamma}^{\prime}(\xi)\right)^{2}\right)^{3 / 2}}\right|>\frac{1}{2^{3 / 2}}
$$

and we get a contradiction to the fact that $H_{\gamma}\left(f_{\gamma}(\xi), \xi\right)$ is arbitrarily close to 0 for $\gamma$ small enough by Step 4 . Therefore the domain of $f_{\gamma}$ can be extended to $\left(x_{2}^{0}-\delta, 1\right]$. Applying the same argument for the left-end point, we find that $f_{\gamma}$ can be defined on the whole interval $[0,1]$. Similarly, the other boundary component $\Sigma_{2, \gamma}$ of $\Omega_{\gamma}$ coincides with the graph of $x_{2} \mapsto 1 / 4+g_{\gamma}\left(x_{2}\right)$ for some smooth function $g_{\gamma}:[0,1] \rightarrow \mathbb{R}$, and the claim follows.

Step 6. Now we will show that for small $\gamma>0$, the minimizers $\left\{u_{\gamma}\right\}$ of $E_{\gamma}$ are lamellar. In light of Step 5, note that the set $\Omega_{\gamma}=\left\{x: u_{\gamma}(x)=1\right\}$ takes the form

$$
S_{1}:=\left\{\left(x_{1}, x_{2}\right) \in \mathbb{T}^{2}: 1 / 4+g_{\gamma}\left(x_{2}\right) \leqslant x_{1} \leqslant 3 / 4+f_{\gamma}\left(x_{2}\right), 0 \leqslant x_{2} \leqslant 1\right\} .
$$

To begin, we note that without loss of generality, we may assume that

$$
\int_{0}^{1} f_{\gamma} \mathrm{d} x_{2}=\int_{0}^{1} g_{\gamma} \mathrm{d} x_{2}=0
$$

Indeed, translating the set $S$ in the $x_{1}$-direction by the amount $\int_{0}^{1} f_{\gamma} \mathrm{d} x_{2}$ and then redefining the graphs $f_{\gamma}$ and $g_{\gamma}$ in terms of the deviation from this new vertical strip, we see that 3.5 follows, in light of the condition $\int_{0}^{1}\left(f_{\gamma}\left(x_{2}\right)-g_{\gamma}\left(x_{2}\right)\right) \mathrm{d} x_{2}=0$ which holds due to the mass constraint $\left|\Omega_{\gamma}\right|=1 / 2$.

Now we will proceed to show that the global minimality of $u_{\gamma}$ is violated for small $\gamma$ unless $u_{\gamma} \equiv u_{L}$. To this end, for $t \in[0,1]$, define

$$
S_{t}:=\left\{\left(x_{1}, x_{2}\right) \in \mathbb{T}^{2}: 1 / 4+\operatorname{tg}_{\gamma}\left(x_{2}\right) \leqslant x_{1} \leqslant 3 / 4+t f_{\gamma}\left(x_{2}\right), 0 \leqslant x_{2} \leqslant 1\right\} .
$$

Note that $\chi_{S_{t}} \rightarrow \chi_{S}$ in $L^{1}$ as $t \rightarrow 0$ and the mass constraint $\int_{\mathbb{T}^{2}} u_{\gamma} \mathrm{d} x=0$ along with 3.5 implies that $\left|S_{t}\right|=1 / 2$ for all $t \in[0,1]$. Hence, the family of functions

$$
U(x, t):=\left\{\begin{array}{ll}
1 & \text { if } x \in S_{t}, \\
-1 & \text { if } x \in S_{t}^{c}
\end{array},\right.
$$

are all admissible competitors in the minimization of $E_{\gamma}$. We then let $V(x, t)$ be the solution of $-\Delta V(\cdot, t)=U(\cdot, t)$ subject to $\int_{\mathbb{T}^{2}} V(x, t) \mathrm{d} x=0$. Note that $U(x, 0)=u_{L}(x), U(x, 1)=u_{\gamma}(x)$, $V(x, 0)=v_{L}(x)$ and $V(x, 1)=v_{\gamma}(x)$.

For $E_{\gamma}(U)(t)=\frac{1}{2} \int_{\mathbb{T}^{2}}|\nabla U(x, t)|+\gamma \int_{\mathbb{T}^{2}}|\nabla V(x, t)|^{2} \mathrm{~d} x$, define

$$
e_{\gamma}(t):=E_{\gamma}(U)(t)
$$


where $e_{\gamma}(0)=E_{\gamma}\left(u_{L}\right)$ and $e_{\gamma}(1)=E_{\gamma}\left(u_{\gamma}\right)$. Taylor's Theorem implies that

$$
e_{\gamma}(1)=e_{\gamma}(0)+e_{\gamma}^{\prime}(0)+\frac{1}{2} e_{\gamma}^{\prime \prime}(\tau)=e_{\gamma}(0)+\frac{1}{2} e_{\gamma}^{\prime \prime}(\tau)
$$

for some $\tau \in(0,1)$ as $u_{L}$ is a critical point of $E_{\gamma}$, making $e_{\gamma}^{\prime}(0)=0$.

Now we are going to calculate $e_{\gamma}^{\prime \prime}(\tau)$ explicitly. Since

$$
\frac{1}{2} \int_{\mathbb{T}^{2}}|\nabla U(x, t)|=\int_{0}^{1}\left(1+t^{2}\left(f_{\gamma}^{\prime}\left(x_{2}\right)\right)^{2}\right)^{1 / 2} \mathrm{~d} x_{2}+\int_{0}^{1}\left(1+t^{2}\left(g_{\gamma}^{\prime}\left(x_{2}\right)\right)^{2}\right)^{1 / 2} \mathrm{~d} x_{2},
$$

a straightforward calculation yields

$$
\begin{aligned}
\frac{\mathrm{d}^{2}}{\mathrm{~d} t^{2}} \frac{1}{2} & \int_{\mathbb{T}^{2}}|\nabla U(x, \tau)| \\
= & \int_{0}^{1}\left\{\left(1+\tau^{2}\left(f_{\gamma}^{\prime}\left(x_{2}\right)\right)^{2}\right)^{-1 / 2}\left(f_{\gamma}^{\prime}\left(x_{2}\right)\right)^{2}+\left(1+\tau^{2}\left(g_{\gamma}^{\prime}\left(x_{2}\right)\right)^{2}\right)^{-1 / 2}\left(g_{\gamma}^{\prime}\left(x_{2}\right)\right)^{2}\right\} \mathrm{d} x_{2} \\
& -\tau^{2} \int_{0}^{1}\left\{\left(1+\tau^{2}\left(f_{\gamma}^{\prime}\left(x_{2}\right)\right)^{2}\right)^{-3 / 2}\left(f_{\gamma}^{\prime}\left(x_{2}\right)\right)^{4}+\left(1+\tau^{2}\left(g_{\gamma}^{\prime}\left(x_{2}\right)\right)^{2}\right)^{-3 / 2}\left(g_{\gamma}^{\prime}\left(x_{2}\right)\right)^{4}\right\} \mathrm{d} x_{2} .
\end{aligned}
$$

Using the fact that $\tau \in(0,1)$ along with the conditions $\left(f_{\gamma}^{\prime}\left(x_{2}\right)\right)^{2}<1$ and $\left(g_{\gamma}^{\prime}\left(x_{2}\right)\right)^{2}<1$ by Step 5, one readily obtains the inequality

$$
\frac{\mathrm{d}^{2}}{\mathrm{~d} t^{2}} \frac{1}{2} \int_{\mathbb{T}^{2}}|\nabla U(x, \tau)|>\frac{1}{2 \sqrt{2}}\left(\int_{0}^{1}\left(f_{\gamma}^{\prime}\left(x_{2}\right)\right)^{2} \mathrm{~d} x_{2}+\int_{0}^{1}\left(g_{\gamma}^{\prime}\left(x_{2}\right)\right)^{2} \mathrm{~d} x_{2}\right) .
$$

Now using the definition of $U(x, t)$ and integrating by parts once, we can rewrite the nonlocal part of the energy as

$$
\begin{aligned}
\int_{\mathbb{T}^{2}}|\nabla V(x, t)|^{2} \mathrm{~d} x & =\int_{\mathbb{T}^{2}} U(x, t) V(x, t) \mathrm{d} x \\
& =\iint_{S_{t}} V\left(x_{1}, x_{2}, t\right) \mathrm{d} x_{1} \mathrm{~d} x_{2}-\iint_{S_{t}^{c}} V\left(x_{1}, x_{2}, t\right) \mathrm{d} x_{1} \mathrm{~d} x_{2} \\
& =\int_{0}^{1} \int_{1 / 4+t g_{\gamma}\left(x_{2}\right)}^{3 / 4+t f_{\gamma}\left(x_{2}\right)} V\left(x_{1}, x_{2}, t\right) \mathrm{d} x_{1} \mathrm{~d} x_{2}-\int_{0}^{1} \int_{3 / 4+t f_{\gamma}\left(x_{2}\right)}^{1 / 4+t g_{\gamma}\left(x_{2}\right)} V\left(x_{1}, x_{2}, t\right) \mathrm{d} x_{1} \mathrm{~d} x_{2} .
\end{aligned}
$$

Then taking two derivatives with respect to $t$, we find

$$
\begin{aligned}
\frac{\mathrm{d}^{2}}{\mathrm{~d} t^{2}} \int_{\mathbb{T}^{2}}|\nabla V(x, \tau)|^{2} \mathrm{~d} x & \\
= & 2 \int_{0}^{1}\left\{V_{x_{1}}\left(3 / 4+\tau f_{\gamma}\left(x_{2}\right), x_{2}, \tau\right) f_{\gamma}^{2}\left(x_{2}\right)-V_{x_{1}}\left(1 / 4+\tau g_{\gamma}\left(x_{2}\right), x_{2}, \tau\right) g_{\gamma}^{2}\left(x_{2}\right)\right\} \mathrm{d} x_{2} \\
& +2 \int_{0}^{1}\left\{V_{t}\left(3 / 4+\tau f_{\gamma}\left(x_{2}\right), x_{2}, \tau\right) f_{\gamma}\left(x_{2}\right)-V_{t}\left(1 / 4+\tau g_{\gamma}\left(x_{2}\right), x_{2}, \tau\right) g_{\gamma}\left(x_{2}\right)\right\} \mathrm{d} x_{2}
\end{aligned}
$$


Let us concentrate on the second integral on the right-hand side of (3.8). Letting $G$ denote the Green's function for the periodic Poisson problem, we get

$$
\begin{aligned}
V\left(3 / 4+t f_{\gamma}\left(x_{2}\right), x_{2}, t\right) & =\iint_{\mathbb{T}^{2}} G\left(3 / 4+t f_{\gamma}\left(x_{2}\right), x_{2}, y_{1}, y_{2}\right) U\left(y_{1}, y_{2}, t\right) \mathrm{d} y_{1} \mathrm{~d} y_{2} \\
& =\left(\iint_{S_{t}}-\iint_{S_{t}^{c}}\right) G\left(3 / 4+t f_{\gamma}\left(x_{2}\right), x_{2}, y_{1}, y_{2}\right) \mathrm{d} y_{1} \mathrm{~d} y_{2} .
\end{aligned}
$$

Similarly we have

$$
V\left(1 / 4+\operatorname{tg}_{\gamma}\left(x_{2}\right), x_{2}, t\right)=\left(\iint_{S_{t}}-\iint_{S_{t}^{c}}\right) G\left(1 / 4+\operatorname{tg} g_{\gamma}\left(x_{2}\right), x_{2}, y_{1}, y_{2}\right) \mathrm{d} y_{1} \mathrm{~d} y_{2} .
$$

Taking the derivative of $V\left(3 / 4+t f_{\gamma}\left(x_{2}\right), x_{2}, t\right)$ and $V\left(1 / 4+t g_{\gamma}\left(x_{2}\right), x_{2}, t\right)$ with respect to $t$ and using the fact that $\int_{\mathbb{T}^{2}} G(x, y) \mathrm{d} y=0$, we obtain

$$
\begin{aligned}
\int_{0}^{1}\left\{V_{t}\left(3 / 4+\tau f_{\gamma}\left(x_{2}\right), x_{2}, \tau\right) f_{\gamma}\left(x_{2}\right)-V_{t}\left(1 / 4+\tau g_{\gamma}\left(x_{2}\right), x_{2}, \tau\right) g_{\gamma}\left(x_{2}\right)\right\} \mathrm{d} x_{2} \\
=4 \int_{0}^{1} \int_{0}^{1}\left\{\begin{array}{l}
G\left(3 / 4+\tau f_{\gamma}\left(x_{2}\right), x_{2}, 3 / 4+\tau f_{\gamma}\left(y_{2}\right), y_{2}\right) f_{\gamma}\left(x_{2}\right) f_{\gamma}\left(y_{2}\right) \\
-G\left(3 / 4+\tau f_{\gamma}\left(x_{2}\right), x_{2}, 1 / 4+\tau g_{\gamma}\left(y_{2}\right), y_{2}\right) f_{\gamma}\left(x_{2}\right) g_{\gamma}\left(y_{2}\right) \\
-G\left(1 / 4+\tau g_{\gamma}\left(x_{2}\right), x_{2}, 3 / 4+t f_{\gamma}\left(y_{2}\right), y_{2}\right) g_{\gamma}\left(x_{2}\right) f_{\gamma}\left(y_{2}\right) \\
\left.+G\left(1 / 4+\tau g_{\gamma}\left(x_{2}\right), x_{2}, 1 / 4+\tau g_{\gamma}\left(y_{2}\right), y_{2}\right) g_{\gamma}\left(x_{2}\right) g_{\gamma}\left(y_{2}\right)\right\} \mathrm{d} x_{2} \mathrm{~d} y_{2} .
\end{array}\right.
\end{aligned}
$$

Hence, the second integral on the right-hand side of the equation $(3.8)$ becomes

$$
4 \int_{\partial S_{\tau}} \int_{\partial S_{\tau}} G(x, y) \zeta(x) \zeta(y) \mathrm{d} \mathcal{H}^{1}(x) \mathrm{d} \mathcal{H}^{1}(y),
$$

where $\zeta$ is defined to be $f_{\gamma}\left(x_{2}\right)$ and $-g_{\gamma}\left(x_{2}\right)$ on the two components of $\partial S_{\tau}$. However, by [13, Chapter 1], we have

$$
\int_{\partial S_{\tau}} \int_{\partial S_{\tau}} G(x, y) \zeta(x) \zeta(y) \mathrm{d} \mathcal{H}^{1}(x) \mathrm{d} \mathcal{H}^{1}(y)=\int_{\mathbb{T}^{2}}|\nabla \omega|^{2} \mathrm{~d} x \geqslant 0,
$$

where $\omega$ is an $H^{1}$ weak solution to the equation

$$
-\Delta \omega=\mu \quad \text { on } \mathbb{T}^{2},
$$

and $\mu$ is the measure given by $\zeta \mathcal{H}^{1}\left\lfloor\partial S_{\tau}\right.$.

Recall that $-\Delta V(x, t)=U(x, t)$, and since $|U|=1$, it follows from elliptic regularity that $\left\|V_{x_{1}}\right\|_{L^{\infty}} \leqslant C_{0}$ for some $C_{0}>0$, independent of $t$ or $\gamma$. Hence, returning to (3.8), and dropping the second integral on the right-hand side, which has been shown to be positive by (3.9), we get

$$
\begin{aligned}
\frac{\mathrm{d}^{2}}{\mathrm{~d} t^{2}} \int_{\mathbb{T}^{2}} & |\nabla V(x, \tau)|^{2} \mathrm{~d} x \\
& \geqslant 2 \int_{0}^{1}\left\{V_{x_{1}}\left(1 / 2+\tau f_{\gamma}\left(x_{2}\right), x_{2}, \tau\right) f_{\gamma}^{2}\left(x_{2}\right)-V_{x_{1}}\left(\tau g_{\gamma}\left(x_{2}\right), x_{2}, \tau\right) g_{\gamma}^{2}\left(x_{2}\right)\right\} \mathrm{d} x_{2} \\
& \geqslant-2 C_{0}\left(\int_{0}^{1} f_{\gamma}^{2}\left(x_{2}\right) \mathrm{d} x_{2}+\int_{0}^{1} g_{\gamma}^{2}\left(x_{2}\right) \mathrm{d} x_{2}\right) .
\end{aligned}
$$


Now combining the equations (3.7) and (3.10) with (3.6), and invoking (3.5) to apply the Poincaré inequality for $f_{\gamma}$ and $g_{\gamma}$, we get

$$
\begin{aligned}
e_{\gamma}(1)= & e_{\gamma}(0)+\frac{1}{2} e_{\gamma}^{\prime \prime}(\tau) \\
\geqslant & e_{\gamma}(0)+\frac{1}{4 \sqrt{2}}\left(\int_{0}^{1}\left(f_{\gamma}^{\prime}\left(x_{2}\right)\right)^{2} \mathrm{~d} x_{2}+\int_{0}^{1}\left(g_{\gamma}^{\prime}\left(x_{2}\right)\right)^{2} \mathrm{~d} x_{2}\right) \\
& -\gamma C_{0}\left(\int_{0}^{1} f_{\gamma}^{2}\left(x_{2}\right) \mathrm{d} x_{2}+\int_{0}^{1} g_{\gamma}^{2}\left(x_{2}\right) \mathrm{d} x_{2}\right) \\
\geqslant & e_{\gamma}(0)+\left(\frac{\pi^{2}}{4 \sqrt{2}}-\gamma C_{0}\right)\left(\int_{0}^{1} f_{\gamma}^{2}\left(x_{2}\right) \mathrm{d} x_{2}+\int_{0}^{1} g_{\gamma}^{2}\left(x_{2}\right) \mathrm{d} x_{2}\right) .
\end{aligned}
$$

For $\gamma<\pi^{2} /\left(4 \sqrt{2} C_{0}\right)$, we conclude from the minimality of $E_{\gamma}\left(u_{\gamma}\right)\left(=e_{\gamma}(1)\right)$ that necessarily $f_{\gamma} \equiv 0 \equiv g_{\gamma}$, that is, $u_{\gamma} \equiv u_{L}$.

\section{Acknowledgments}

This research was supported by the National Science Foundation DMS-0654122. The authors would like to thank Rustum Choksi, Jiri Dadok, Chuck Livingston, Cyrill Muratov, Bruce Solomon and Yoshi Tonegawa for helpful conversations. They are also grateful to Umberto Massari for pointing out to them Lemma 2.1 of [7] which plays a crucial role in the proof of regularity.

\section{REFERENCES}

1. CHOKSI, R. Nonlocal Cahn-Hilliard and isoperimetric problems: Periodic phase separation induced by competing long- and short-term interactions. In: Singularities in PDE and the Calculus of Variations, CRM Proc. Lecture Notes, Amer. Math. Soc., Providence (2008), 33-45. Zbl pre05296078 MR 2528732

2. Choksi, R., \& Peletier, M. A. Small volume fraction limit of the diblock copolymer problem I: Sharp interface functional. SIAM J. Math. Anal. 42 (2010), 1334-1370. MR 2653253

3. Choksi, R., Peletier, M. A., \& Williams, J. F. On the phase diagram for microphase separation of diblock copolymers: An approach via a nonlocal Cahn-Hilliard functional. SIAM J. Appl. Math. 69 (2009), 1712-1738. Zbl pre05653776 MR 2496714

4. Choksi, R., \& Sternberg, P. Periodic phase separation: the periodic Cahn-Hilliard and isoperimetric problems. Interfaces Free Bound. 8 (2006), 371-392. Zbl 1109.35092 MR 2273234

5. CHOKSi, R., \& STERnBerg, P. On the first and second variations of a nonlocal isoperimetric problem. J. Reine Angew. Math. 611 (2008), 75-108. Zbl 1132.35029 MR 2360604

6. Gilbarg, D., \& Trudinger, N. S. Elliptic Differential Equations of Second Order. Springer (1998).

7. GiUsti, E. The equilibrium configuration of liquid drops. J. Reine Angew. Math. 321 (1981), 53-63. Zbl 0438.76078 MR 0597979

8. GIUSTI, E. Minimal Surfaces and Functions of Bounded Variation. Birkhäuser (1984). Zbl 0545.49018 MR 0775682

9. Grosse-Brauckmann, K. Stable constant mean curvature surfaces minimize area. Pacific J. Math. 175 (1997), 527-534. Zbl 0878.49026 |MR 1432843

10. hauswirth, L., Pérez, J., Romon, P., \& Ros, A. The periodic isoperimetric problem. Trans. Amer. Math. Soc. 356 (2004), 2025-2047. Zbl 1046.52002 MR 2031051 
11. Hiroshima, T. Construction of the Green function on a Riemannian manifold using harmonic coordinates. J. Math. Kyoto Univ. 36 (1996), 1-30. Zbl 0867.58066 MR 1381536

12. Howards, H., Hutchings, M., \& Morgan, F. The isoperimetric problem on surfaces. Amer. Math. Monthly 106 (1999), 430-439. Zbl 1003.52011 MR 1699261

13. Landkof, N. S. Foundations of Modern Potential Theory. Springer (1972). Zbl 0253.31001 MR 0350027

14. MASSARI, U. Esistenza e regolarità delle ipersuperfici di curvatura media assegnata in $\mathbb{R}^{n}$. Arch. Ration. Mech. Anal. 55 (1974), 357-382. Zbl 0305.49047 MR 0355766

15. Montero, J., Sternberg, P., \& Ziemer, W. Local minimizers with vortices to the GinzburgLandau system in 3d. Comm. Pure Appl. Math. 57 (2004) 99-125. Zbl 1052.49002 MR 2007357

16. Morgan, F., \& Ros, A. Stable constant-mean-curvature hypersurfaces are area minimizing in small $L^{1}$ neighborhoods. Interfaces Free Bound. 12 (2010), 151-155. Zbl 1195.49055 MR 2652015

17. Muratov, C. B., Droplet phases in non-local Ginzburg-Landau models with Coulomb repulsion in two dimensions. Comm. Math. Phys. 299 (2010), 45-87. Zbl pre05789929 MR 2672798

18. ОнтA, T., \& KAWASAKI, K. Equilibrium morphology of block copolymer melts. Macromolecules 19 (1986), 2621-2632.

19. Peletier, M. A., \& Veneroni, M. Stripe patterns in a model for block copolymers. Math. Models Methods Appl. Sci. 20 (2010), 843-907. Zbl 1203.49018 MR 2659741

20. REN, X., \& WEI, J. On the multiplicity of two nonlocal variational problems. SIAM J. Math. Anal. 31 (2000), 909-924. Zbl 0973.49007 MR 1752422

21. REN, X., \& WEI, J. Concentrically layered energy equilibria of the diblock copolymer problem. Eur. J. Appl. Math. 13 (2002), 479-496. Zbl 1010.82041 MR 1939157

22. REN, X., \& WEI, J. On the spectra of three dimensional lamellar solutions of the diblock copolymer problem. SIAM J. Math. Anal. 35 (2003), 1-32. Zbl 1055.35041 MR 2001463

23. REN, X., \& WEI, J. Stability of spot and ring solutions of the diblock copolymer equation. J. Math. Phys. 45 (2004), 4106-4133. Zbl 1064.82040 MR 2098120

24. REN, X., \& WEI, J. Wriggled lamellar solutions and their stability in the diblock copolymer problem. SIAM J. Math. Anal. 37 (2005), 455-489. Zbl 1136.35372 MR 2176111

25. REN, X., \& WEI, J. Many droplet pattern in the cylindrical phase of diblock copolymer morphology. Rev. Math. Phys. 19 (2007), 879-921. Zbl 1145.82007 MR 2349026

26. REN, X., \& WEI, J. Spherical solutions to a nonlocal free boundary problem from diblock copolymer morphology. SIAM J. Math. Anal. 39 (2008), 1497-1535. Zbl 1153.35091 MR 2377287

27. REN, X., \& WEI, J. Oval shaped droplet solutions in the saturation process of some pattern formation problems. SIAM J. Math. Anal. 70 (2009), 1120-1138. Zbl 1198.35009 MR 2546355

28. RÖGER, M., \& TONEGAWA, Y. Convergence of phase field approximations to the Gibbs-Thomson law. Calc. Var. 32 (2008), 111-136. Zbl 1159.49046 MR 2377408

29. Sternberg, P., \& Ziemer, W. Local minimizers of a three-phase partition problem with triple junctions. Proc. Roy. Soc. Edinburgh Sect. A 124 (1994), 1059-1073. Zbl 0843.49025 MR 1313189

30. TAmanini, I. Boundaries of Caccioppoli sets with Hölder-continuous normal vector. J. Reine Angew. Math. 334 (1982), 27-39. Zbl 0479.49028 MR 0667448

31. Wehrheim, K. Uhlenbeck Compactness. Eur. Math. Soc. (2004). Zbl 1055.53027| MR 2030823 